\title{
AVALIAÇÃO DA VELOCIDADE DE RESFRIAMENTO SOBRE A MICROESTRUTURA E RESISTÊNCIA A CORROSÃO DE UM AÇO DUPLEX*
}

\author{
Maurício dos Santos Vasconcelos ${ }^{1}$ \\ Rômulo Mendes de Barros ${ }^{1}$ \\ Gabrielle Laut Lopes Marinho ${ }^{1}$ \\ Monique Osório Talarico da Conceição ${ }^{2}$ \\ Mônica Silva Araújo Freire ${ }^{3}$ \\ Wilma Clemente de Lima Pinto ${ }^{4}$ \\ Ana Isabel de Carvalho Santana ${ }^{5}$
}

Resumo

Os aços inoxidáveis são normalmente aplicados em ambientes de elevada agressividade devido à combinação de propriedades, tais como resistência mecânica e química. Os aços duplex são um tipo especial de aços inoxidáveis que devido à sua microestrutura bifásica apresentam propriedades que possibilitam sua aplicação em diferentes meios: indústria química, petroquímica, de petróleo e gás, nuclear entre outras. Contudo, devido a condições operacionais e de processo podem acarretar na formação de fases/precipitados $(\sigma, \chi$, nitretos, carbetos etc). A fase sigma $(\sigma)$, é considerada uma das principais responsáveis pela diminuição das propriedades mecânicas e da resistência à corrosão dos aços duplex. Considerando a importância da aplicação dos aços duplex, muitos trabalho podem ser encontrados na literatura acerca da relação entre a temperatura e a precipitação dessas fases,. Dessa forma este trabalho tem como objetivo realizar uma avaliação do efeito da velocidade de resfriamento sobre a microestrutura e a resistência à corrosão do aço duplex em meio ácido.

Palavras-chave: Aço duplex; Microestrutura; Corrosão

\section{EVALUATION OF THE COOLING SPEED ON THE MICROSTRUCTURE AND CORROSION RESISTANCE OF A DUPLEX STEEL}

\begin{abstract}
Stainless steels are usually applied in environments of high aggressiveness due to the combination of properties such as mechanical and chemical resistance. Duplex steels are a special kind of stainless steels that, due to their biphasic microstructure, have properties that allow their application in different environments: chemical, petrochemical, oil and gas, and nuclear industries. However, due to operational and process conditions, the formation of precipitates $(\sigma, \chi$, nitrides, carbides, etc.) may occur. The sigma phase $(\sigma)$ is one of the main factors responsible for the reduction of the mechanical properties and corrosion resistance of duplex steels. Considering the importance of duplex steels aplications, many papers can be found in the literature on the relationship between temperature and precipitation of these phases. In this way, this work aims to evaluate the effect of the cooling rate on the microstructure and the corrosion resistance of the duplex steel in the acid medium.
\end{abstract}

Keywords: Duplex steel; Microstructure; Corrosion

(Aluno de iniciação), Laboratório de Eletroquímica e Microscopia de Materiais, CSPI, Fundação Centro Universitário Estadual da Zona Oeste - UEZO, Rio de Janeiro/RJ, Brasil.

2 Física, Doutorado, professora, Centro Universitário de Volta Redonda - UNIFOA, Volta Redonda/RJ;

3 Engenheira Metalurgista, Pós-graduanda - Universidade Pitágoras Unopar, Brasil.

4 Engenheira Química, Doutorado, professora Adjunto, Laboratório de Eletroquímica e Microscopia de Materiais, CSPI, Fundação Centro Universitário Estadual da Zona Oeste - UEZO, Rio de Janeiro/RJ, Brasil

5 Química, Doutorado, professora Adjunto, Laboratório de Eletroquímica e Microscopia de Materiais, CSPI, Fundação Centro Universitário Estadual da Zona Oeste - UEZO, Rio de Janeiro/RJ, Brasil. 


\section{INTRODUÇÃO}

Os aços inoxidáveis são exemplos de ligas ferrosas frequentemente utilizadas quando o meio ambiente apresenta condições agressivas, que exigem do material metálico utilizado uma combinação de propriedades, tais como resistência mecânica e química. Dentro da classificação dos aços inox, existe uma classe especial, denominada aço inoxidável duplex que vem sendo frequentemente aplicados em meios de elevada agressividade: indústria química, petroquímica, de petróleo e gás, nuclear entre outras. A ampla aplicação dos aços classificados como duplex, super e hiper duplex, se deve à combinação de boas propriedades mecânicas, elevada resistência a corrosão e soldabilidade [1 - 3].

Contudo, apesar da elevada resistência à corrosão desses materiais, podem ocorrer situações que provocam um aumento da susceptibilidade à corrosão, em virtude da precipitação de fases, partículas deletérias (fases sigma, chi, nitretos, carbetos $\mathrm{M}_{23} \mathrm{C}_{6}$, austenita secundária) [4,5]. A fase sigma (s), é considerada uma das principais responsáveis pela diminuição das propriedades mecânicas e da resistência à corrosão dos aços duplex, em virtude da sua rápida cinética de formação.

De uma forma geral, processos que envolvem transferência de calor tais como procedimentos de soldagem, processamento a quente e aplicações desses materiais em temperaturas elevadas, podem provocar a precipitação dessas fases secundárias [4,5]. Dessa forma é de fundamental importância compreender a relação entre a temperatura e o tempo de processos a quente, com a precipitação de fases e a consequente perda de propriedades desses materiais.

Considerando a importância da aplicação dos aços duplex, bem como a relação custo/benefício desses materiais para o meio industrial, este trabalho tem como objetivo realizar uma avaliação do efeito da velocidade de resfriamento em amostras de aços duplex termicamente tratadas, e a relação com a microestrutura e a resistência à corrosão dessas amostras.

\section{MATERIAIS E MÉTODOS}

O material utilizado neste trabalho consiste em uma amostra de aço inoxidável duplex cuja composição química está descrita na tabela 1.

Tabela 1. Composição química em peso (\%)

\begin{tabular}{cccccccccc}
\hline $\mathbf{C}$ & $\mathbf{S i}$ & $\mathbf{M n}$ & $\mathbf{P}$ & $\mathbf{S}$ & $\mathbf{~ N i}$ & $\mathbf{C r}$ & $\mathbf{M o}$ & $\mathbf{C u}$ & $\mathbf{F e}$ \\
\hline 0,050 & 0,480 & 0,750 & 0,036 & 0,009 & 4,04 & 22,50 & 3,65 & 0,192 & balanço \\
\hline
\end{tabular}

As amostras metálicas foram cortadas em cortadeira metalográfica de bancada (TECLAGO, modelo xxxx). Após o corte, as amostras foram submetidas a tratamento térmico de solubilização a $1050^{\circ} \mathrm{C}$, por um período de trinta, seguido de resfriamento em água. Após solubilização as amostras foram termicamente tratadas a uma temperatura de $800^{\circ} \mathrm{C}$ por um período de vinte minutos e submetidas a três diferentes tipos de resfriamento. Parte das amostras foi resfriada em água, parte resfriada ao ar, e parte resfriada em forno. Dessa forma, ao final do tratamento térmico obteve-se cinco diferentes amostras do aço duplex: amostra como recebida $(\mathrm{CR})$, amostra solubilizada (SOL), amostra solubilizada, termicamente tratada e resfriada em água (TT- ÁGUA), amostra solubilizada, termicamente tratada e 
resfriada ao ar (TT - AR) e amostra solubilizada, termicamente tratada e resfriada em forno (TT - FORNO).

Após tratamento térmico, os corpos de prova, foram preparados para análise da microestrutura. Inicialmente as amostras foram lixadas em lixa d'água com granulometria $(80,120,220,400,600,1200)$ com auxílio de uma lixadeira de bancada (AROTEC, modelo Aropol VV). Após foram polidas em pano de polimento e pasta diamente com granulometria de 3 e $1 \mu \mathrm{m}$, lavadas com água destilada, desengorduradas com álcool e secas com jato de ar frio. Em seguida as amostras foram quimicamente atacadas com reagente Behara modificado $\left(\mathrm{H}_{2} \mathrm{O}+\mathrm{HCl}+\right.$ $\mathrm{K}_{2} \mathrm{~S}_{2} \mathrm{O}_{5}$ ). $\mathrm{O}$ ataque foi realizado mediante imersão das amostras lixadas e polidas na solução Behara, por tempo suficiente para distinção das fases.

A microestrutura das amostras foi avaliada através de microscopia ótica, utilizando microcopio ótico (OLYMPUS, modelo BX51M) e por microscopia eletrônica de varredura, através do microscópio de varredura (JEOL).

A análise do efeito do tratamento térmico sobre a resistência a corrosão das amostras foi realizada mediante ensaios eletroquímicos. Para realização dos ensaios, foi realizado o contato elétrico das amostras metálicas, com um fio de cobre, em seguida as amostras foram embutidas em resina epóxi e curadas por um período de 72 horas. Antes dos testes eletroquímicos, as amostras foram lixadas em lixa d'água (100, 200, 300, 400 e 600 mesh), lavadas com água e álcool e secas com jato de ar frio.

Os ensaios eletroquímicos foram realizados utilizando uma célula de três eletrodos (duplex - eletrodo de trabalho, platina - eletrodo auxiliar, calomelano eletrodo de referência). Todos os ensaios eletroquímicos foram conduzidos em meio eletrolítico constituído de solução $0,1 \mathrm{M}$ de $\mathrm{HCl}$, aerado e a temperatura ambiente. Os ensaios foram obtidos por meio de um potenciostato/galvanostato (AUTOLAB, PGSTAT 302N). As curvas de polarização foram obtidas após estabilização do potencial de circuito aberto, durante $1800 \mathrm{~s}$, no modo potenciodinâmico, com intervalo de potencial entre $-1 \mathrm{~V}$ a $1,5 \mathrm{~V}$ e velocidade de varredura de $1 \mathrm{mV} / \mathrm{S}$.

\section{RESULTADOS E DISCUSSÃO}

As Figuras 1 a 5, apresentam as micrografias obtidas para as amostras de aço duplex nas diferentes condições (como recebida, solubilizada e termicamente tratada com diferentes condições de resfriamento). Observa-se que as amostras na forma como recebida e solubilizada (Figuras 1 e 2) apresentam a microestrutura clássica, onde se verifica as fases ferrítica - $\alpha$ (fase clara - matriz), e a fase austenita - $\gamma$ (fase escura). Tanto na amostra como recebida quanto na solubilizada, verifica-se que a fase $\gamma$ se encontra disposta no formato de "ilhas" ao longo da fase $\alpha$. Não se verificou a precipitação de outras fases nestas condições. Observa-se que para a amostra solubilizada a fase austenita apresenta grãos menos alongados em comparação com a amostra como recebida.

É possível verificar que após o tratamento térmico a $800^{\circ} \mathrm{C}$ (Figuras $3-5$ ) observou-se além das fases ferrita e austenita, a presença de precipitados formados na região do contorno de grão das fases $\gamma / \alpha$ e dentro da matriz $\alpha$. A formação dos precipitados é observada para as amostras submetidas aos três diferentes tipos de resfriamento (água, ar e forno). Através das micrografias obtidas por microscopia 
ótica observa-se que os precipitados (fase branca) sobretudo na região do contorno de grão. Através da microscopia eletrônica de varredura, os precipitados podem ser mais facilmente verificados. Observa-se que a velocidade do resfriamento, influencia diretamente na quantidade dos precipitados formados. As amostras resfriadas em forno (resfriamento lento) apresentam um maior número de precipitados em comparação com as amostras resfriadas ao ar e em água. Um comportamento semelhante vem sendo observado na literatura, onde se observa que precipitados tais como carbonetos $\left(\mathrm{M}_{23} \mathrm{C}_{6}\right)$, nitretos $\left(\mathrm{Cr}_{2} \mathrm{~N}\right)$, fase $\sigma$ e fase $\chi$ são formados ao longo do contorno de grão e na matriz ferrítica em função da temperatura e do tempo do tratamento térmico. Yang e colaboradores [4], assim como Zhang e colaboradores [1] reportaram a precipitação da fase $\sigma$ e de carbonetos para amostras tratadas num intervalo de temperatura entre $700-850^{\circ} \mathrm{C}$, em diferentes tempos de tratamento.

Considerando os resultados obtidos e as condições experimentais aplicadas neste trabalho, é possível sugerir que o tratamento térmico realizado $\left(800^{\circ} \mathrm{C}, 20\right.$ minutos) provocou a precipitação da fase $\sigma$ no contorno de grão das fases ferrita/austenita. Essa precipitação é mais significativa para a amostra resfriada em forno, pois em virtude da baixa velocidade de resfriamento a amostra permanece por mais tempo na faixa de temperatura que promove a precipitação da fase sigma.
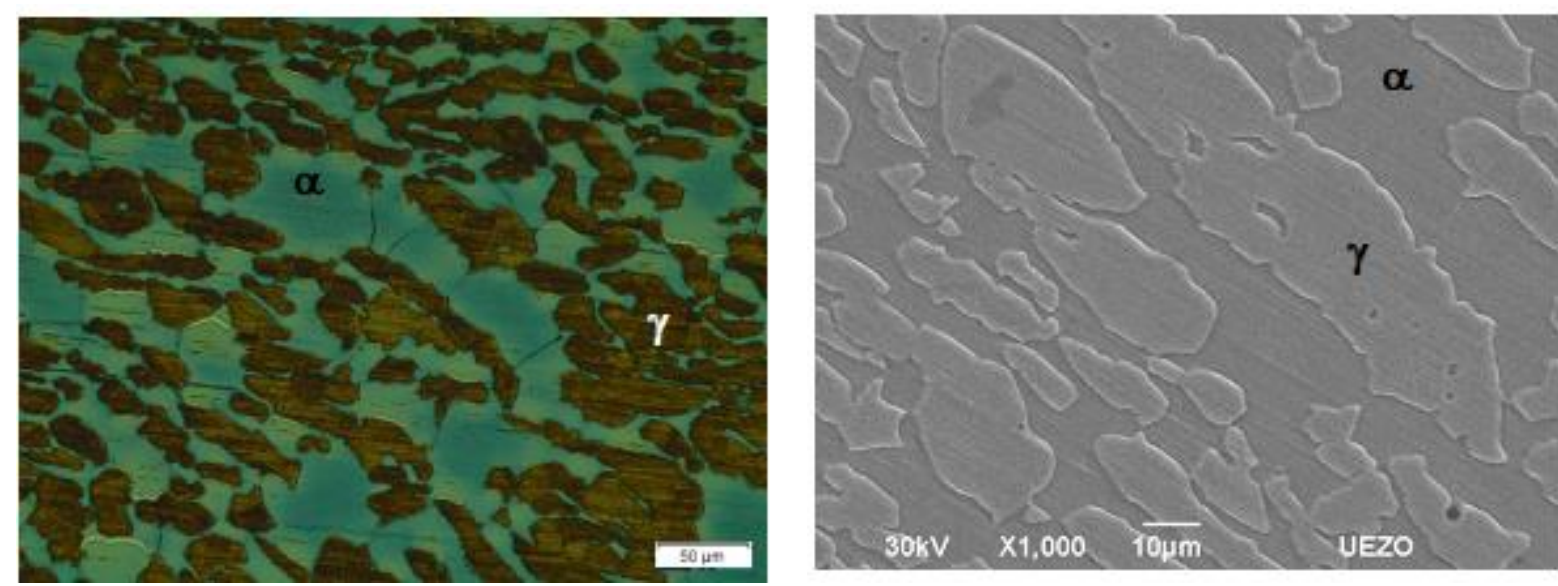

Figura 1. Micrografias obtidas para a amostra como recebida, ataque Behara modificado: microscopia ótica e microscopia eletrônica de varredura.
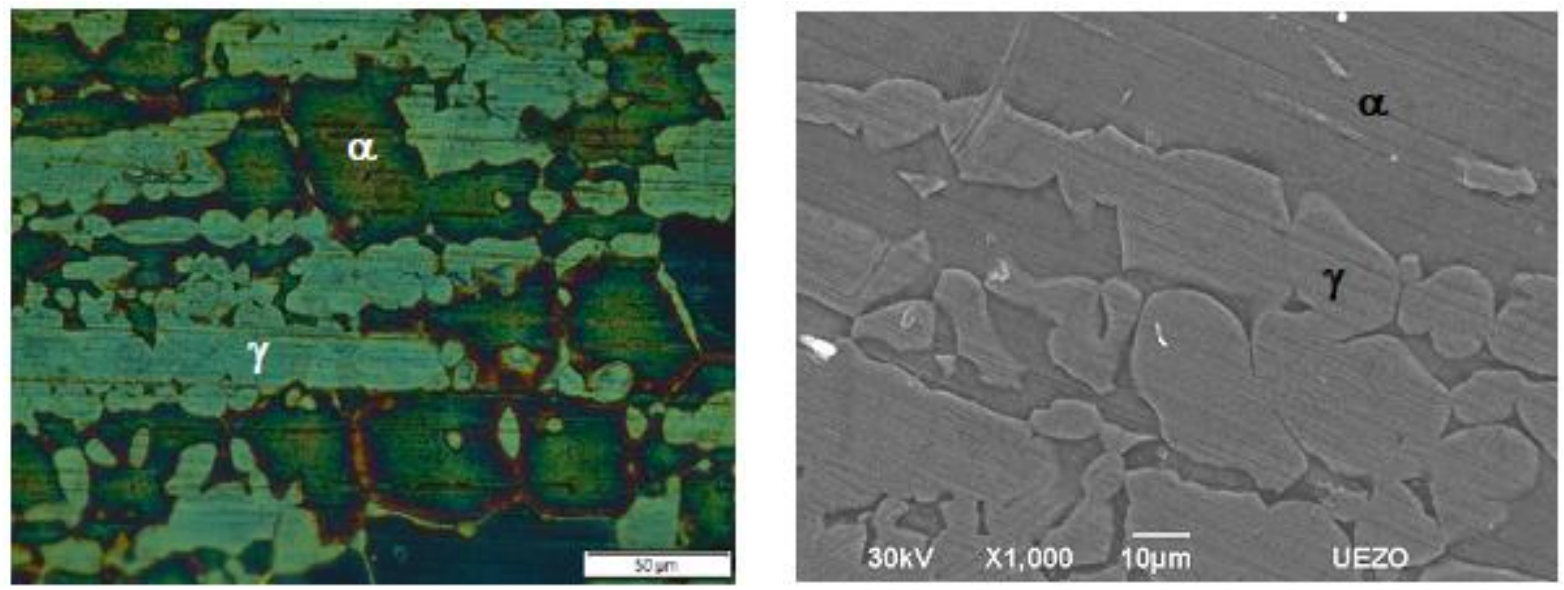

Figura 2. Micrografias obtidas para a amostra solubilizada (SOL), ataque Behara modificado: microscopia ótica e microscopia eletrônica de varredura. 

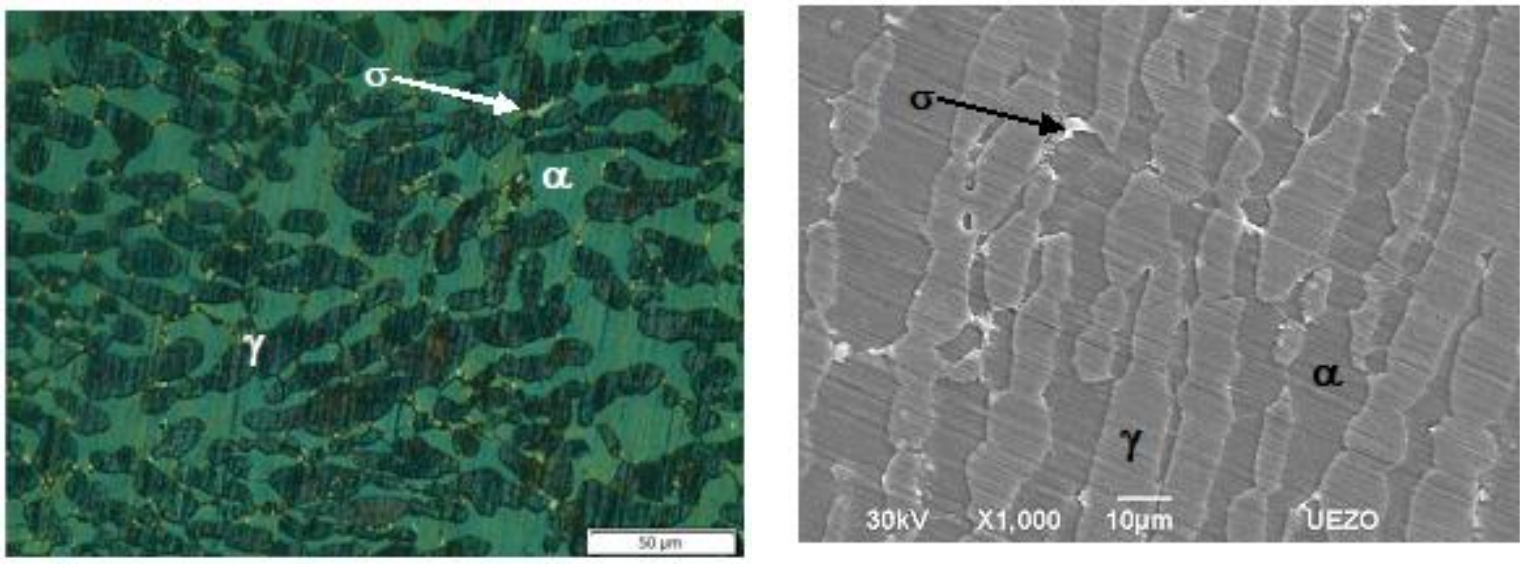

Figura 3. Micrografias obtidas para a amostra solubilizada, termicamente tratada e resfriada em água (TT - ÁGUA), ataque Behara modificado: microscopia ótica e microscopia eletrônica de varredura.
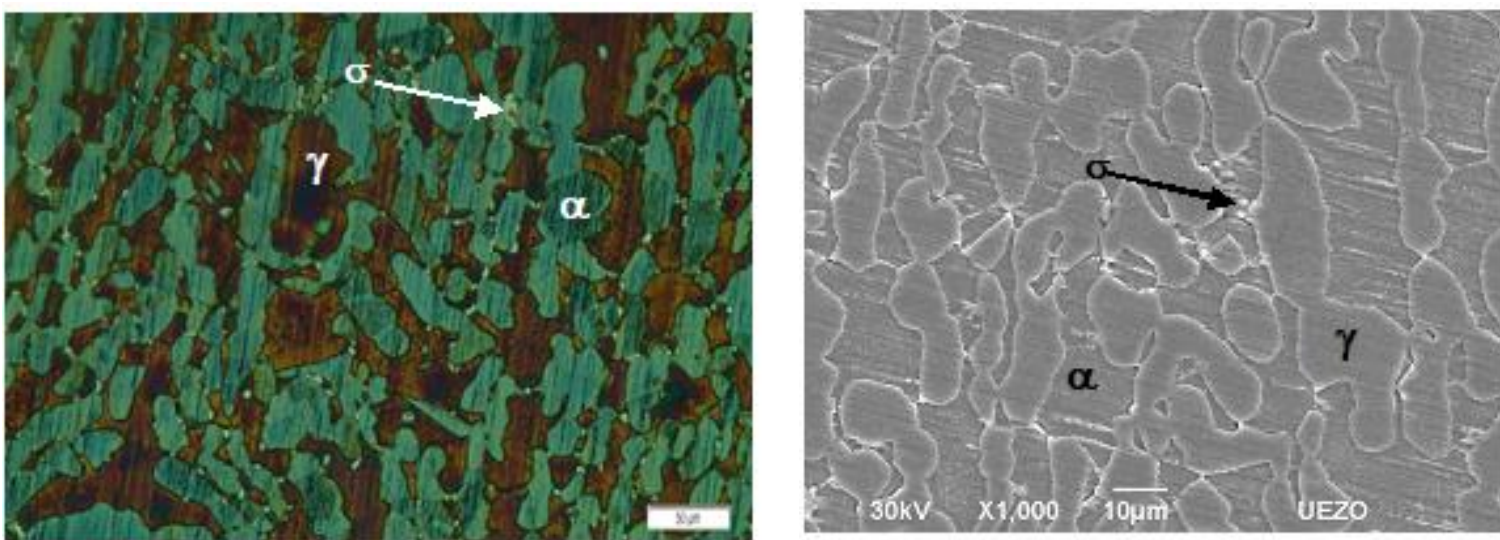

Figura 4. Micrografias obtidas para a amostra solubilizada, termicamente tratada e resfriada em ar (TT - AR), ataque Behara modificado: microscopia ótica e microscopia eletrônica de varredura.
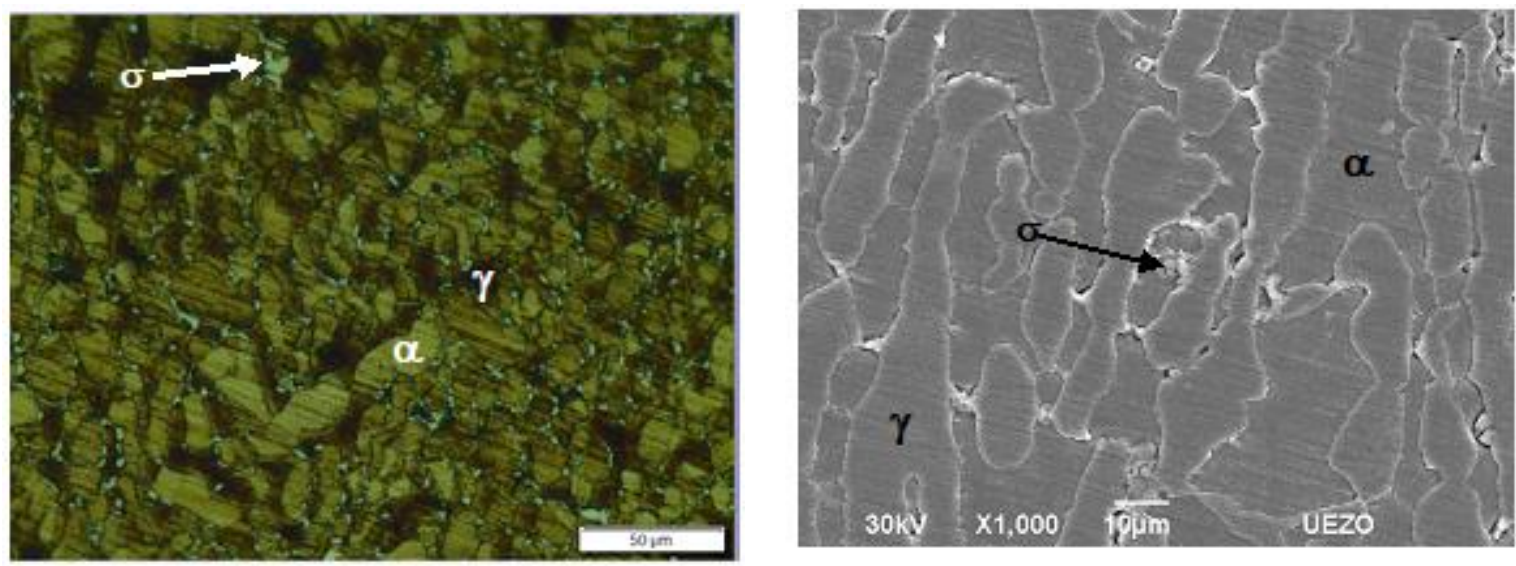

Figura 5. Micrografias obtidas para a amostra solubilizada, termicamente tratada e resfriada em ar (TT - AR), ataque Behara modificado: microscopia ótica e microscopia eletrônica de varredura.

A relação entre a precipitação da fase sigma e a resistência a corrosão das amostras metálicas pode ser verificada através das curvas de polarização 
potenciodinâmicas (Figura 6) obtidas para as amostras nas diferentes condições de tratamento térmico. Inicialmente não foram observadas diferenças significativas para as amostras como recebida, solubilizada, bem como para as amostras termicamente tratadas resfriadas em água e ao ar. Nestas condições todas as amostras apresentaram uma região passiva considerável, e a corrente de passivação é muito próxima para as amostras citadas. Da mesma forma, não se observou diferenças no potencial de corrosão e no potencial de pite, uma vez que todas amostras citadas apresentam a quebra do filme de passivação por volta de $800 \mathrm{mV}$.

Por outro lado, a amostra termicamente tratada, resfriada em forno, apresenta um comportamento completamente diferente das demais. Neste caso não se observa região passiva, a corrente apresenta um aumento contínuo em toda escala de potencial avaliada. Esse comportamento está diretamente relacionado à maior quantidade de fase sigma $(\sigma)$, precipitada ao longo do contorno de grão. As amostras resfriadas ao ar e em água não apresentam comportamento similar, porque em virtude da elevada taxa de resfriamento, a quantidade de fase sigma precipitada não foi suficiente para sensitizar a amostra, ou seja, para diminuir a resistência a corrosão nas condições experimentais avaliadas.

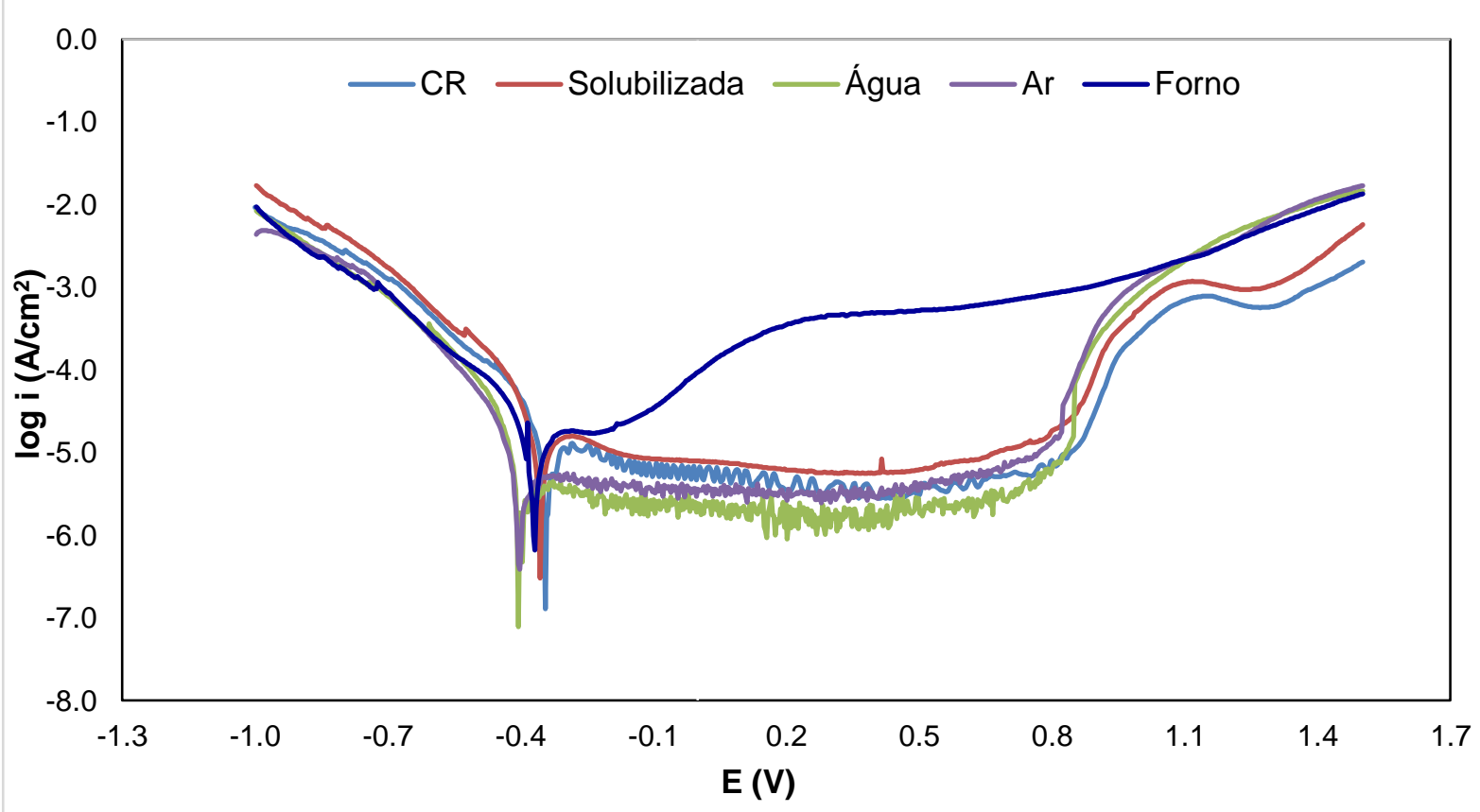

Figura 6. Curvas de polarização obtidas para as amostras de aço duplex, nas diferentes condições de tratamento térmico, em $0,1 \mathrm{M}$ de $\mathrm{HCl}$, meio aerado a temperatura ambiente.

\section{CONCLUSÃO}

Considerando os resultados obtidos é possível concluir que processos que envolvam temperatura de operação por volta de $800^{\circ} \mathrm{C}$, podem provocar a precipitação de fases que acarretarão em perdas de importantes propriedades para o material. A velocidade do resfriamento tem uma significativa influência sobre a 
precipitação das fases. Verificou-se que em resfriamentos mais lentos, ocorre um aumento da precipitação da fase sigma.

Os ensaios eletroquímicos mostraram que a precipitação de fase sigma foi significativa para as amostras tratadas em forno. Neste caso ocorreu uma considerável diminuição na resistência a corrosão das amostras resfriadas em forno, em comparação com as amostras submetidas a outras condições de tratamento térmico.

\section{Agradecimentos}

Agradecemos a FAPERJ pelo apoio concedido.

\section{REFERÊNCIAS}

1 Zhang Z, Zhao H, Zhang H, Yu Z, Hu J, He L, Li J. Effect of isothermal aging on the pitting corrosion resistance of UNSS82441 duplex stainless steel based on electrochemical detection, Corrosion Science. 2015; 93: 120 - 125.

2 Kim H-J, Jeon S-H, Kim S-T, Lee I-S, Park Y-S, Kim K-T, Kim Y-S. Investigation of the sensitization and intergranular corrosion of tube-to-tubesheet welds of hyper duplex stainless steel using an electrochemical reactivation method, Corrosion Science. 2014; 87: 60 - 70.

3 Leiva-García R, Fernandes JCS, Muñoz-Portero MJ, García-Antón J, Study of the sensitisation process of a duplex stainless steel (UNS1.4462) by means of confocal microscopy and localised electrochemical techniques, Corrosion Science. 2015; 94: $327-341$.

4 Yang, YH, Yan B, Wang J, Yin JL, The influence of solution treatment temperature on microstructure and corrosion behavior of high temperature ageing in $25 \% \mathrm{Cr}$ duplex stainless steel, Journal of Alloys and Compounds. 2011; 509: $8870-8879$.

5 Jeon S-H, Kim H-J, Park Y-S, Effects of inclusions on the precipitation of chi phases and intergranular corrosion resistance of hyper duplex stainless steel, Corrosion Science. 2014; 87: 1 - 5. 